Article

\title{
Line of Sight Correction of High-Speed Liquid Crystal Using Overdriving Technology
}

\author{
Hongyang Guo ${ }^{1,2,3} \mathbb{D}$, Qing $\mathrm{Li}^{1,2,3,4}$, Yangjie $\mathrm{Xu}{ }^{1,2,3}$, Yongmei Huang ${ }^{1,2,3}$ and \\ Shengping Du 1,2,3,*
}

1 Key Laboratory of Optical Engineering, Chinese Academy of Sciences, No.1 Guangdian Road, Chengdu 610209, China; guohongy93@163.com (H.G.); Qingli@163.com (Q.L.); yangjie@163.com (Y.X.); yongmei@163.com (Y.H.)

2 The Institute of Optics and Electronics, Chinese Academy of Sciences, Chengdu 610209, China

3 School of Optoelectronics, University of Chinese Academy of Sciences, Beijing 100049, China

4 School of Optoelectronic Science and Engineering, University of Electronic Science and Technology of China, No. 4 Section 2 North Jianshe Road, Chengdu 610054, China

* Correspondence: du_shengping@163.com; Tel.: +86-186-0801-1989

Received: 4 August 2020; Accepted: 7 September 2020; Published: 10 September 2020

\begin{abstract}
In the line of sight correction system, the response time of the liquid crystal spatial light modulator under the normal driving voltage is too long to affect system performance. On the issues, an overdriving method based on a Field-Programmable Gate Array (FPGA) is established. The principle of the overdrive is to use a higher voltage difference to achieve a faster response speed of liquid crystal. In this scheme, the overdriving look-up table is used to seek the response time of the quantized phase, and the liquid crystal electrode is driven by Pulse-Width Modulation (PWM). All the processes are performed in FPGA, which releases the central processing unit (CPU) memory and responds faster. Adequate simulations and experiments are introduced to demonstrate the proposed method. The overdriving experiment shows that the rising response time is reduced from $530 \mathrm{~ms}$ to $34 \mathrm{~ms}$, and the falling time is from $360 \mathrm{~ms}$ to $38 \mathrm{~ms}$ under the overdriving voltage. Typical light tracks are imitated to evaluate the performance of the line of sight correction platform. Results show that using the overdrive the $-3 \mathrm{~dB}$ rejection frequency was increased from $1.1 \mathrm{~Hz}$ to $2.6 \mathrm{~Hz}$. The suppression ability of the overdrive is about $-20 \mathrm{~dB}$ at $0.1 \mathrm{~Hz}$, however the normal-driving suppression ability is only about $-13 \mathrm{~dB}$.
\end{abstract}

Keywords: liquid crystal; overdrive; FPGA; response time; diffraction

\section{Introduction}

Liquid crystal spatial light modulator (LCSLM) uses liquid crystal layers as light modulation materials. Different electric fields are applied to change the alignment direction and position of the liquid crystal molecules. Finally, the optical properties are changed to realize the modulation of the optical signal. LCSLM has become one of the hottest topics in the field of adaptive optics, free space optical, beam deflection and beam phased array in recent years. Because of the electrically controlled birefringence, LCSLM can modulate light amplitude or phase by generating different path patterns, without moving parts [1-3]. Furthermore, driven by an electric field, low power consumption and micro-radius level resolution [4-6], making LCSLM an ideal device of spatial line of sight correction.

However, the application of LCSLM light correction technology in laser communication is still in an experimental demonstration stage. The slow response speed limited the system bandwidth $[7,8]$. The common method to improve the speed is by synthesizing fast response materials or using new materials [9-11], but the potential is limited. In view of the slow response of LCSLM under normal 
driving voltage, Wu Shicong [12] first proposed an overdriving method [13]. The basic principle is based on using a higher voltage difference to achieve a faster response speed, which means increasing the basic voltage of the circuit to drive the LCSLM. This method further improves the response speed on the basis of liquid crystal materials. Since then, domestic and international scholars have carried out a lot of research on overdriving technology. For the liquid crystal display, the switching speed for the Boulder Nonlinear systems $512 \times 512$ pixel SLM filled with nematic liquid crystal for visible wavelengths is less than $100 \mathrm{~ms}$ [14]. In 2006, Xiaodong Xun applied overdriving technology to reduce the rising response time from $340 \mathrm{~ms}$ to $67 \mathrm{~ms}$ and the falling time from $420 \mathrm{~ms}$ to $98 \mathrm{~ms}$ successfully [14]. In 2013, the Graphics Processing Unit (GPU) was used to overdrive the liquid crystal in the adaptive optics, which had produced the rapid correction of far-field distortion spots [15].

However, there are some problems that need to be solved. In terms of data processing, most of the existing overdrive uses the specialized hardware to drive data conversion, and it must be matched with a unique LCSLM. The overdriving lookup table is built in the Central Processing Unit (CPU) or GPU. However, the liquid crystal has megapixels, and each pixel corresponds to a lookup table, which occupies a large amount of CPU memory and slows down the respond speed. In terms of systems, the overdriving method has not been applied to the LCSLM spatial line of sight correction system, and the influence of overdrive has not been analyzed and demonstrated.

Therefore, in this paper, liquid crystal line of sight correction system based on the overdrive will be thoroughly discussed. The first section elaborates the principle of LCSLM and overdriving technology. Secondly, the overdrive system based on Field-Programmable Gate Array (FPGA) is analyzed. Finally, the line of sight correction system based on overdrive is implemented and verified experimentally.

\section{Principle of Overdriving Technology}

\subsection{Relaxation Characteristics of Liquid Crystal}

Nematic Liquid Crystal is shown in Figure 1. The z-axis is normal to the plane of the substrates, and the electric field $\mathrm{E}$ is along the $z$-axis. There is a rotation of molecules director when the electric field is applied to the LCSLM. After the external force is removed, the director returns to the initial state under the intermolecular interaction force. The deflection of the molecular director takes a long time. This process is called relaxation, including dielectric relaxation and steering relaxation. As the typical value of dielectric relaxation is $10^{-4}-10^{-6} \mathrm{~s}$, it has little effect on response time if the frame frequency is $1 \mathrm{kHz}$. We will mainly discuss turning relaxation.

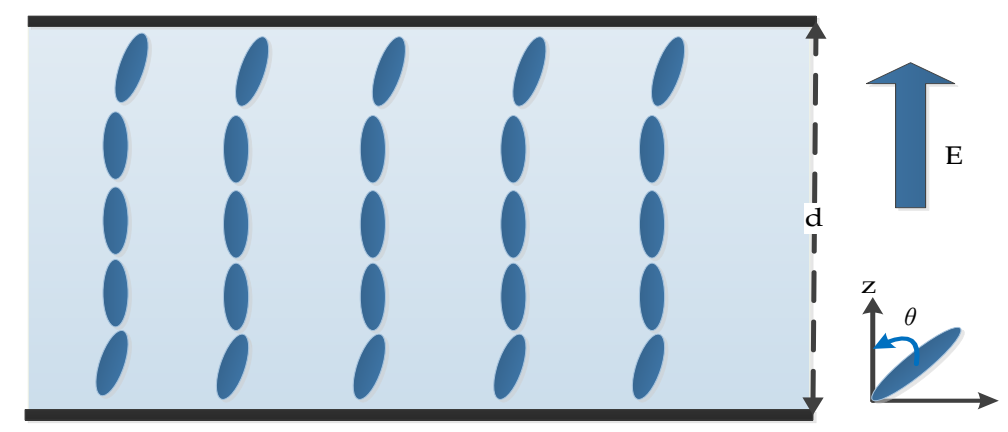

Figure 1. Schematic drawing of a Nematic Liquid Crystal cell.

According to the liquid crystal molecular continuum theory, the distribution function $\theta(x)$ of the director angle, as shown in the following, satisfies the differential equation under the action of electric field and elastic energy [16].

$$
K \frac{\partial^{2} \theta}{\partial z^{2}}+\Delta \varepsilon E^{2} \sin \theta \cos \theta=0
$$


where $K$ is the elastic distortion constant, $E$ is the electric field intensity and $\Delta \varepsilon$ is the anisotropy of dielectric constant. If time is infinite, Equation (1) is stability, and $\theta$ is constant which means $\frac{\partial \theta}{\partial t}=0$. However, the liquid crystal molecules will not turn immediately at the time electric field added, causing $\frac{\partial \theta}{\partial t} \neq 0$. Equation (1) should be modified as following [16]:

$$
K \frac{\partial^{2} \theta}{\partial z^{2}}+\Delta \varepsilon E^{2} \sin \theta \cos \theta=\eta \frac{\partial \theta}{\partial t}
$$

In the equation, $\eta$ is viscosity coefficient of liquid crystal molecules turning. the first item is elastic deformation, the second is the torgue of electric field, and the third is the effect of the liquid crystal viscosity. If the electric field is not more than the Frederiks threshold, the liquid crystal molecular deflection angle is very small. Neglecting the higher harmonics of the molecular vertical distribution function, the relaxation time of steering can be written as follows:

$$
\eta \frac{\partial \theta}{\partial t}=K \frac{\partial^{2} \theta}{\partial z^{2}}+\Delta \varepsilon E^{2} \theta
$$

Equation (3) has following general solution [17]:

$$
\theta=\theta_{m} \cos (\pi z / d) \exp (t / \tau)
$$

where $\theta_{m}$ represents the maximum tilt angle in the center of the liquid crystal cell, $d$ is the cell thickness, $z$ is the vertical direction of cell, $t$ is the response time, and $\tau$ is time constant.

The process, which removes the electric field, is called the falling process. When the electric field just disappears, liquid crystal molecules rotate rapidly. Then the process of back to the initial state is decaying. According to Equation (3), the distribution function $\theta(x)$ of falling process is shown as follows:

$$
\eta \frac{\partial \theta}{\partial t}=K \frac{\partial^{2} \theta}{\partial z^{2}}
$$

The falling time constant $\tau_{f}$ is expressed as [16]:

$$
\tau_{f}=\tau_{0}=\frac{\eta d^{2}}{\pi^{2} K}
$$

where, $\tau_{0}$ is called free relaxation time.

Under the electric field, the rising time constant $\tau_{r}$ is expressed as follows [16]:

$$
\tau_{r}=\frac{\gamma_{1}}{\Delta \varepsilon E^{2}-K \pi^{2} / d^{2}}=\frac{\tau_{0}}{\left(\frac{V}{V_{\text {th }}}\right)^{2}-1}
$$

where, $V_{t h}$ is the threshold voltage. We deduced that the relaxation time can be decreased by reducing the viscosity coefficient or thickness of the liquid crystal box, but the electro-optical properties might be wasted and limited by materials. Therefore, a better method is to appropriately increase the driving voltage within the LCSLM driving range. The phase modulation of the liquid crystal follows the diffraction field theory and changes periodically. The falling time constant is related to the intrinsic property. When the phase modulation exceeds $2 \pi$, the falling response speed can be improved by selecting the fast falling segment of the phase modulation interval.

\subsection{Overdriving Theory}

The principle of the overdriving method is that by using higher voltage difference a faster switching speed can be achieved. It drives the LCSLM with an addressing voltage that exceeds the steady-state 
that is required. When the target phase is reached, the voltage is reset to the steady-state $[18,19]$. The phase is well modeled by the function for the rising case:

$$
\varphi(t)=\varphi_{0}+\left(\varphi_{1}-\varphi_{0}\right)\left(1-\exp \frac{t-t_{0}}{\tau}\right)
$$

where, $\varphi_{0}$ is the initial phase modulation at time $t_{0}, \varphi_{1}$ is the target phase of time $t_{1}$ and $\tau$ is the time constant.

As shown in Figure $2, U_{0}$ is the initial driving voltage, and the modulated phase is $\varphi_{0}$. The objective phase $\varphi_{1}$ is matched with $U_{1}$. The response process will follow the curve 1 , that $\varphi_{1}$ is arrived at the time $t_{2}$. Under the overdriving voltage $U_{2}$, as shown as the curve two, the target phase $\varphi_{1}$ will be arrived at time $t_{1}$. Then we change the driving voltage into $U_{1}$ to maintain the target phase $\varphi_{1}$. In summary, in order to achieve overdriving method, the phase modulation characteristics and the overdriving time look-up table of the liquid crystal are needed.

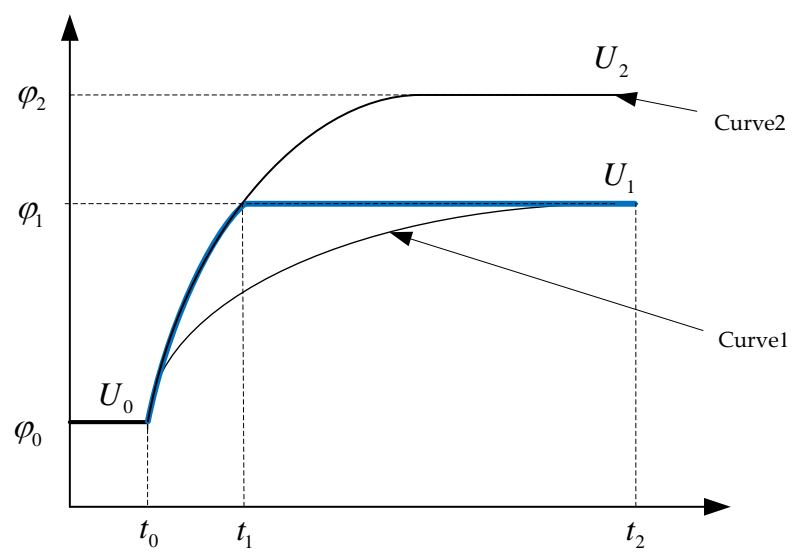

Figure 2. Schematic diagram of overdriving principle.

\section{Implementation of the Overdrive}

\subsection{Measurement of Phase Modulation Characteristics}

Nematic liquid crystal is a uniaxial crystal, which has a birefringent effect. The incident light is modulated to change the optical path difference between the ordinary (o light) and the extraordinary light (e light). For the o light, the refractive index does not change. But for the e light, the refractive index is related to the angle between the light and the director. Therefore, by controlling the driving voltage to modulate the director, the refractive index of e light can be controlled. Finally the birefringence of the liquid crystal can be changed. The refractive index of the e light and the o light propagating in the liquid crystal molecule can be expressed by the refractive index ellipsoid, whose long axis of the molecule serves as the axis of rotation. It can be deduced from theory that the phase difference caused by the change of refractive index is written as [20]:

$$
\Delta \varphi=\frac{4 \Delta V}{V_{t h}}\left(n_{/ /}-n_{\perp}\right) \frac{d}{\lambda}
$$

where, $n_{/ /}$is the length of the ellipsoid axis along the rotation direction, $n_{\perp}$ is the axis length along the vertical rotation direction, $\lambda$ is the wavelength, and $d$ is the liquid crystal cell thickness.

The intensity measured on the photo detector is assumed to be modeled as [21]:

$$
I_{0}=\frac{1+\cos \varphi}{2} E_{0}^{2}
$$


In Equation (10), $I_{0}$ represents the zeroth order intensity, $\varphi$ is the phase shift of the LCSLM pixels and $E_{0}^{2}$ is the zeroth-order light intensity. The diffraction intensity at zero-order is shown as Figure 3.

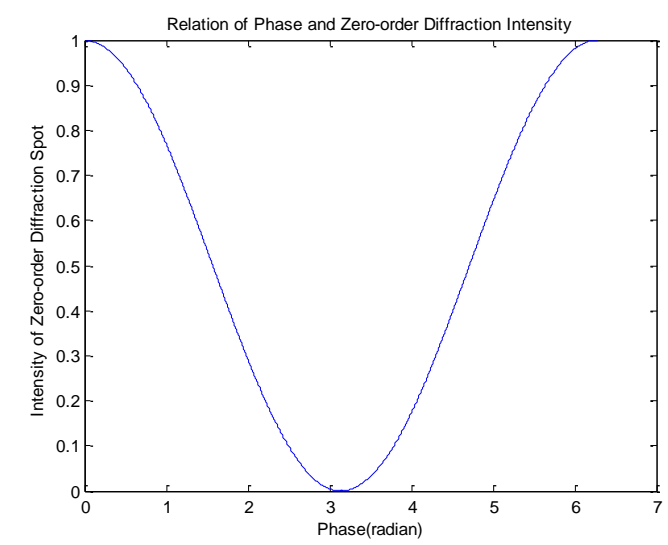

Figure 3. Relationship of phase and zero-order diffraction intensity.

According to the analysis above, the LCSLM modulates the light by applying a different driving voltage and the modulation phase can be obtained by measuring the intensity of the far-field zero-order diffraction spot. Therefore the relationship between the liquid crystal modulation phase and the driving voltage can be obtained by measuring the light intensity change.

\subsection{FPGA Implementation of Overdriving System}

The overdriving system based on the FPGA is shown in Figure 4, including a frame buffer, phase quantizer, overdriving lookup table, lookup table of phase modulation and time controller. In the FPGA, each electrodes previous frame data are cached in the Random Access Memory (RAM) corresponding address to achieve the frame buffer. FPGA reads the previous frame when the computer writes the current frame. Then the initial phase and target phase are obtained. The specific implementation is as follows:

(1) Phase quantizer, a RAM includes an initial value in FPGA, divides $2 \pi$ into an N segment in lookup table. It converts the input two sets of phase into an eight-bit look-up table address, where the upper four-bits represent the initial phase and the lower four-bits represent the target phase.

(2) Overdriving lookup table is the key technology of the system. The module mainly includes three parts: an address generation module, a lookup table and a timing controller. The row and column address of the lookup table is generated according to the current frame phase and the previous frame. The overdriving lookup table is two-dimensional, which is composed of an initial phase, target phase, and response time. In this paper, an overdriving lookup table is implemented in the FPGA with a RAM loading the initial value. The quantization segment is set to an address space for an eight-bit overdriving table. For example, when the address line is 00001000 , the start phase is 0 .

(3) Lookup table of phase modulation is segmented into different intervals. The interval search is performed according to the input phase and the driving electrode duty ratio. The Pulse-Width Modulation (PWM) control duty is used to control the driving voltage, and different duty ratios correspond to different phase modulation intervals.

(4) The time controller keeps the phase and control signal synchronous after overdrive. The signal delay part adopts the D flip-flop series connection, which is equivalent to a shift register. Each clock cycle is passed backwards one level, and the shift output is performed after eight clock cycles. 


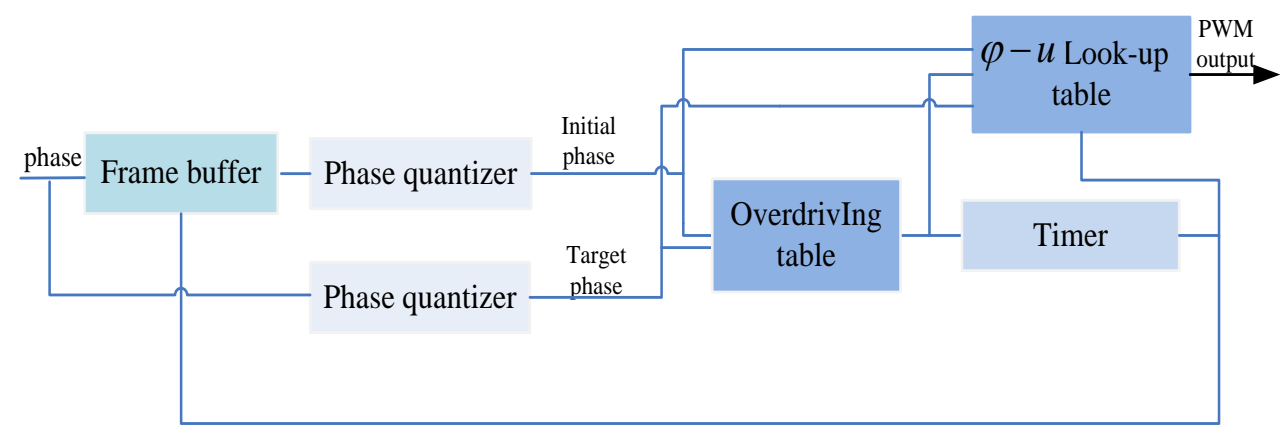

Figure 4. Implementation of Field-Programmable Gate Array overdriving system.

\section{Experimental Verification}

\subsection{Overdriving Experiment}

In the experiment, a pure phase transmitted Nematic liquid crystal developed by the University of Electronic Science and Technology in China is used. The specific parameters are shown in Table 1. It has 1920 electrodes, in which the width is $3 \mu \mathrm{m}$, space is $2 \mu \mathrm{m}$ and the grating constant is 20 . The experimental optical path is shown in Figure 5. The light source is a $671 \mathrm{~nm}$ wavelength laser, which passes through a collimator and polarizer (P) and incident on the LCSLM. After being modulation the light is focused on the Position Sensitive Detector (PSD). The analog output of the PSD is translated to a digital signal and sent to a computer to store and process.

Table 1. Parameters of Nematic liquid crystal. LCSLM: Liquid crystal spatial light modulator.

\begin{tabular}{cc}
\hline Parameters & LCSLM(NT7708) \\
\hline Wavelength range & $620-720 \mathrm{~nm}$ \\
electrode & 1920 \\
Active area & $10 \mathrm{~mm} \times 10 \mathrm{~mm}$ \\
Pixel pitch & $5.0 \mu \mathrm{m}$ \\
Thickness of box & $10 \mu \mathrm{m}$ \\
Damage Threshold (voltage) & $15 \mathrm{~V}$ \\
\hline
\end{tabular}

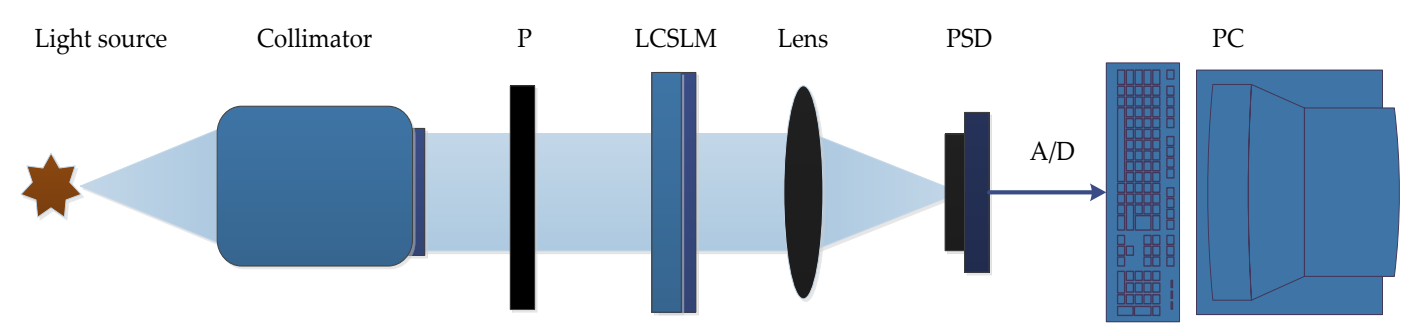

Figure 5. Experimental optical path of overdrive. P: Polarizer, PSD: Position Sensitive Detector, A/D: Analog to Digital Converter, PC: Personal Computer.

The maximum driving voltage we used in the experiment is set to $5 \mathrm{~V}$ in order to ensure the stable operation and better response effect. The PWM duty ratio is divided into 255 . The driving voltage corresponding to zero duty cycle is $0 \mathrm{~V}$, and the 255 duty cycle is $5 \mathrm{~V}$. Rising the PWM duty ratio from zero to 255 . The far-field zero-order spot light intensity is obtained by the PSD. According to the relationship between the driving voltage and the modulation phase in Section 3.1, the electro-optical curve is shown in Figure 6. It can be seen that in the case of the $5 \mathrm{~V}$ driving voltage, the modulation depth of the LCSLM is about $3.5 \pi$. 


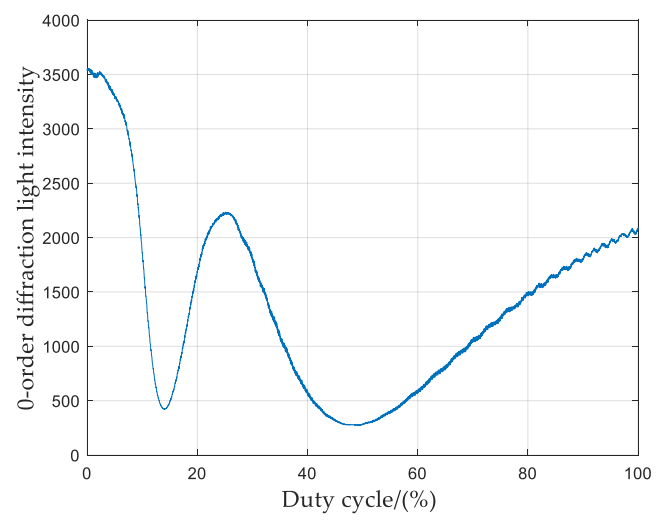

(a)

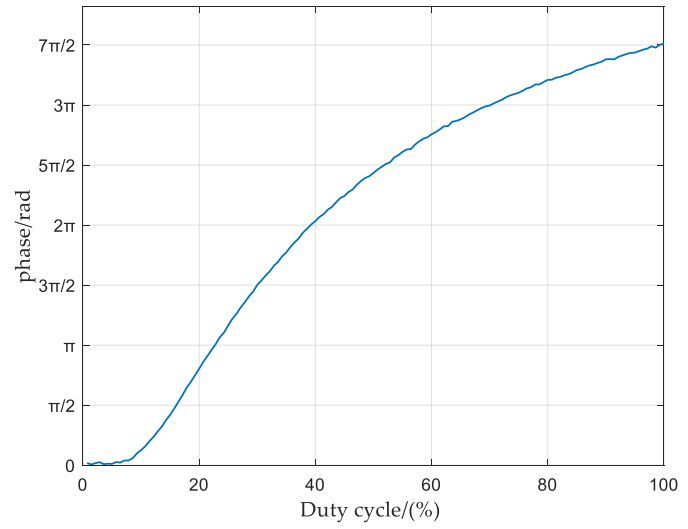

(b)

Figure 6. Phase modulation characteristics of (a) light intensity with duty cycle and (b) modulation phase with duty cycle.

Next, we measure the response time of the LCSLM. For the $2 \pi$ modulation, the PWM duty ratio is about 102, and the corresponding driving voltage is $2 \mathrm{~V}$, which is regarded as the normal driving voltage. During the rising process, the timer starts timing when a $2 \mathrm{~V}$ driving voltage is applied to the LCSLM. At the same time, the light intensity of the 0th order diffraction spot is measured. When the modulation phase reaches $2 \pi$, the timing ends. Similarly, during the falling process, the initial modulation phase of the LCSLM is $2 \pi$, and the driving voltage jumps from $2 \mathrm{~V}$ to $0 \mathrm{~V}$. As the dotted lines shown in Figure 7, the rising response time is about $530 \mathrm{~ms}$ and the falling is about $360 \mathrm{~ms}$.

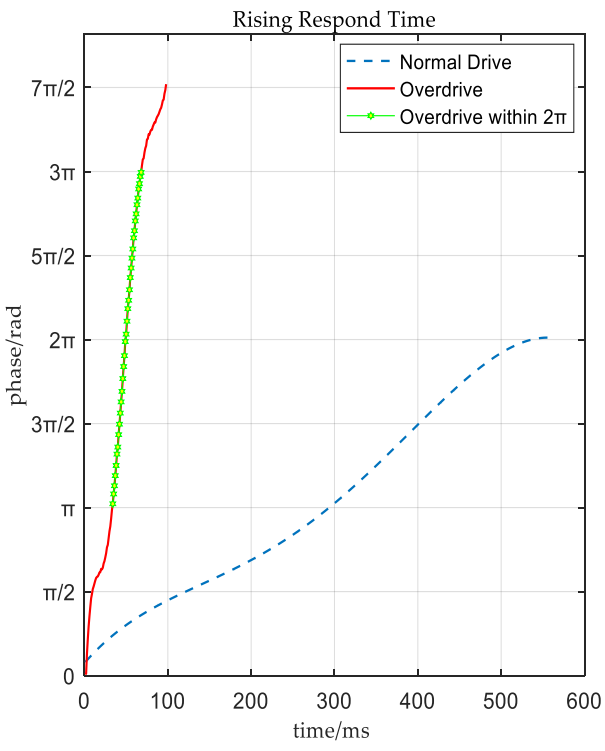

(a)

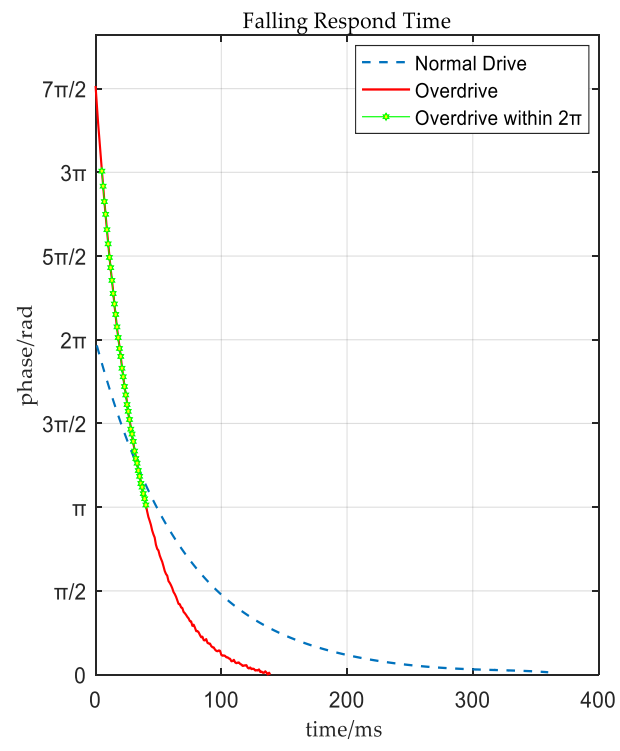

(b)

Figure 7. Response time of (a) the rising process and (b) the falling process with different driving voltage.

According to the overdriving method, the upper limit of the drive voltage is increased to $5 \mathrm{~V}$. The response curve is as the solid lines shown in Figure 7. According to the above analysis, the modulation phase of NT7708 LCSLM exceeds $2 \pi$, we can select a continuous $2 \pi$ phase as the actual modulation phase. The modulation segment is selected to $\pi-3 \pi$, the corresponding PWM is $58-178$. The rising time is $34 \mathrm{~ms}$ and the falling time is reduced to $38 \mathrm{~ms}$, as the square shaped line in Figure 7 . 
It proves that the response speed of the LCSLM is improved by nearly an order of magnitude based on the overdriving technology.

\subsection{System Performance of Line of Sight Correction}

The line of sight correction system is shown in Figure 8. The LCSLM is used as a corrector. The light source is disturbed by the scanning mirror, and the controller loads the correction information onto the LCSLM to track the disturbance target. Analysis and discussion of the sensor and liquid crystal phased array is the basis of the system. The LCSLM is described as a one-order inertial link and pure time delay. The former is mainly caused by the relaxation characteristics of the molecules, and the latter is the delay of driving circuit and the PSD.

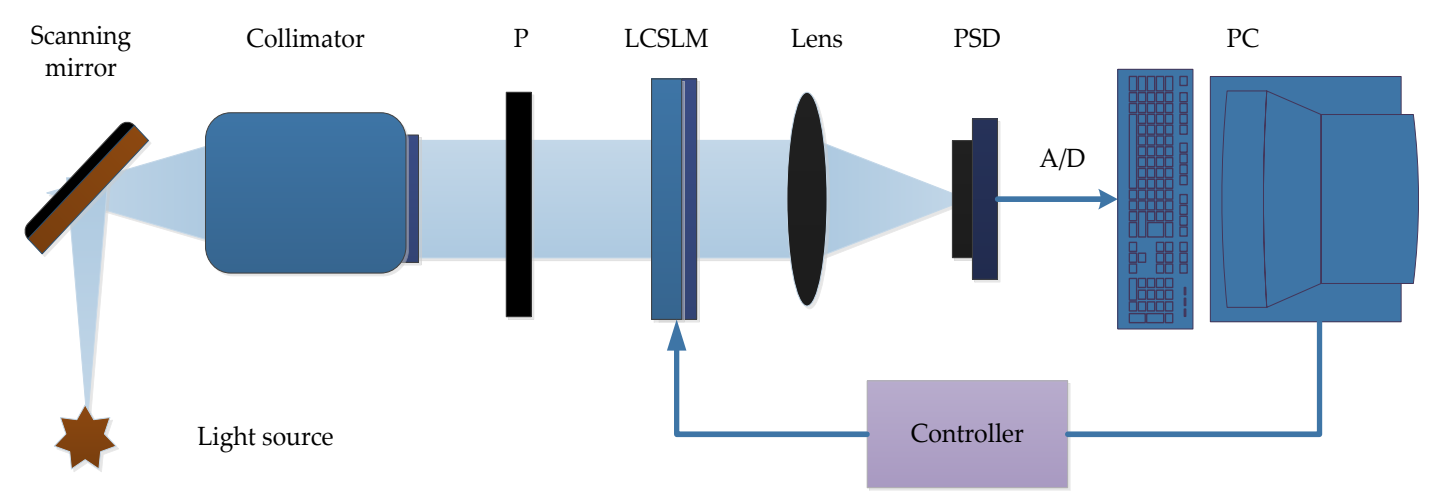

Figure 8. Schematic diagram of line of sight correction system.

The transfer function of the liquid crystal phased array can be expressed as follow:

$$
\mathrm{G}_{\mathrm{obj}}(\mathrm{s})=\frac{1}{1+T_{1} s} e^{-\left(T_{2}+T_{3}\right) s}
$$

where, $T_{1}$ is the delay of the relaxation characteristics of liquid crystal molecules, $T_{2}$ represents the total delay of the LCSLM, $T_{3}$ is the delay of PSD. According to the experimental measurement, the characteristic of LCSLM is fitted as follows:

$$
\mathrm{G}_{\mathrm{obj}}(\mathrm{s})=\frac{5.6}{1+0.03 \mathrm{~s}} e^{-0.10 \mathrm{~s}}
$$

In order to improve the gain of the control system, a proportional integral (PI) closed-loop controller is used to design the system. PI controller is a linear controller that forms a control deviation based on a given value and an actual output value, and linearly combines the proportion and integral of the deviation to form a control quantity to control the controlled object. The closed-loop characteristics of the correction system are obtained under different driving methods as shown as follow.

It can be seen from Figure 9 that the dotted line represents normal drive, and its closed-loop bandwidth is about $1.1 \mathrm{~Hz}$. The overdriving condition represented by the solid line has a smaller delay, and the closed loop bandwidth is exceeding $2.6 \mathrm{~Hz}$. For the above-mentioned, the different error suppression characteristics of the system are obtained, as shown in Figure 10. The error suppression capability of the system at $0.1 \mathrm{~Hz}$ is elevated about $7 \mathrm{~dB}$. 

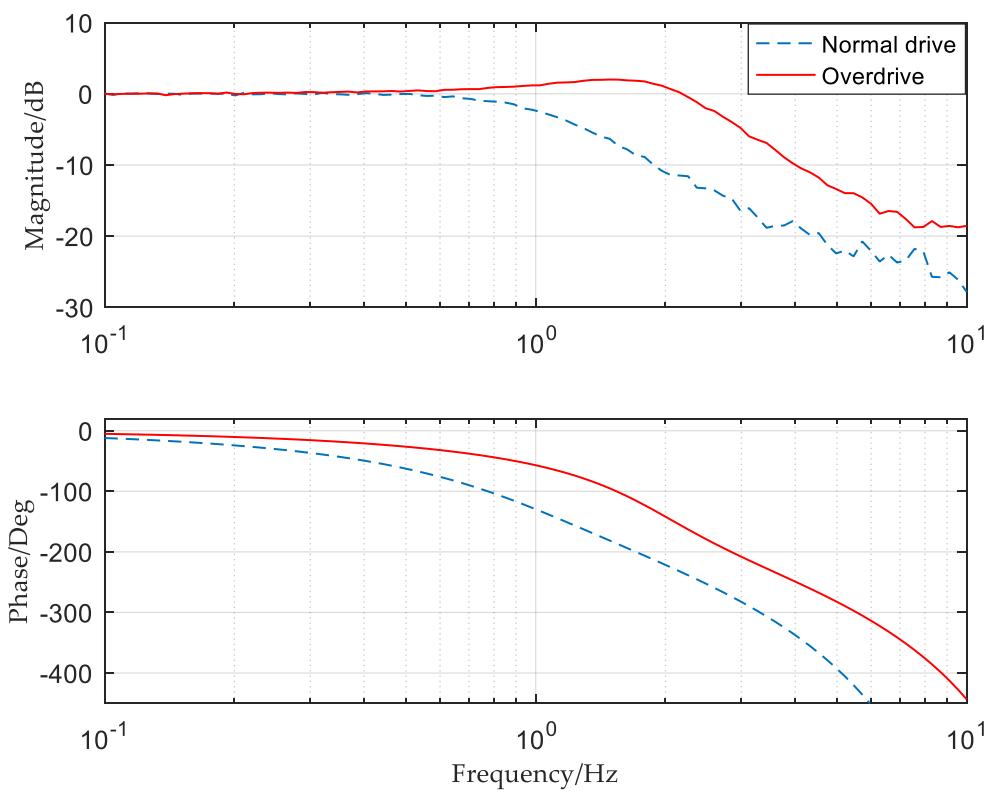

Figure 9. Closed loop characteristics under different driving conditions.
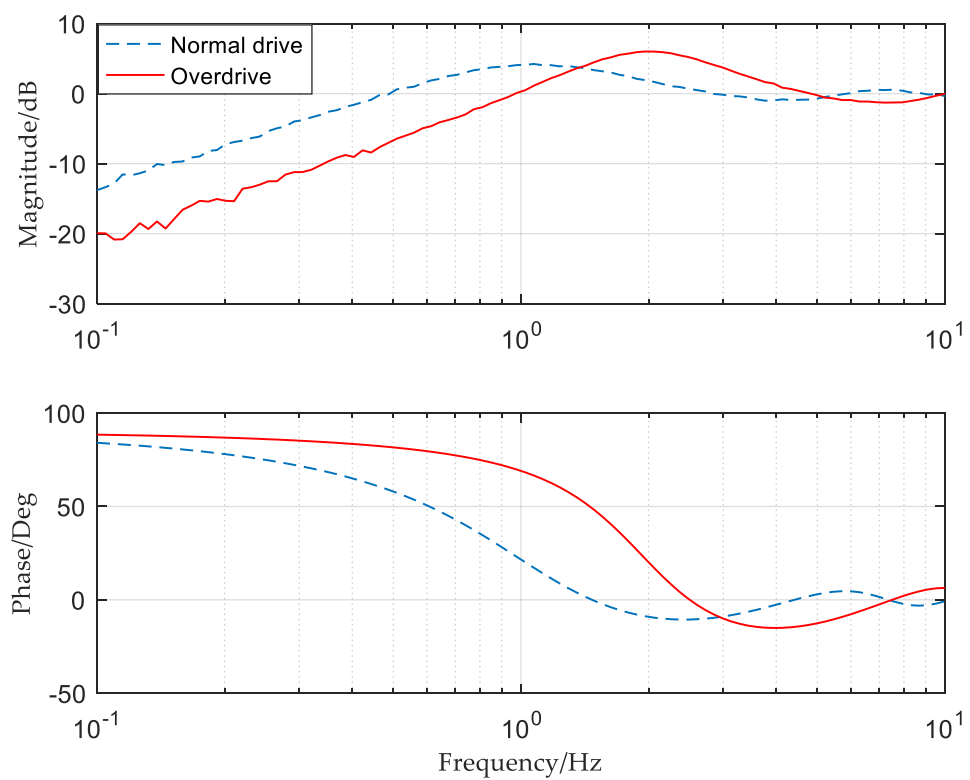

Figure 10. Characteristics of error suppression under different driving conditions.

Through theoretical analysis and experiments, it can be seen that the retardation of the LCSLM has an important influence on the line of sight correction system. Using the overdriving method, the delay of the LCSLM is reduced, and the closed-loop bandwidth is increased, thereby the error suppression capability is improved.

\section{Conclusions}

In this paper, an overdriving scheme based on FPGA is proposed for the slow response of the LCSLM. The experimental results show that the method increases the response speed of liquid crystal by about an order of magnitude under the overdriving voltage.

Compared with the traditional overdrive, all the driving processes are implemented in the FPGA. The driving voltage is controlled by the PWM, the optimal modulation interval is selected reasonably, and the overdriving lookup table is established effectively. Using RAM to search the driver table 
directly does not occupy CPU memory, and reduces CPU response time. By using the overdriving method, the delay of LCSLM is reduced, and the closed-loop bandwidth is increased, thereby the error suppression capability is improved. It has obvious advantages and application significance for adaptive optics and beam deflection, which require a large amount of calculation and a fast response.

Under the existing experimental platform conditions, the delay of the LCSLM and the sensor without overdriving technology is about $270 \mathrm{~ms}$, and the delay under the overdriving technology is approximately $100 \mathrm{~ms}$. According to the theoretical calculation, the sensor delay is about $50 \mathrm{~ms}$, and the LCSLM with overdriving technology still has a delay of $72 \mathrm{~ms}$, which is mainly caused by the electrical driver and the liquid crystal material. The next step is to further reduce the delay of the sensor and improve the performance of the LCSLM.

Author Contributions: Conceptualization, H.G. and S.D.; Methodology, H.G.; software, S.D.; Validation, H.G., Q.L. and Y.X.; Formal analysis, H.G.; Investigation, H.G.; Resources, S.D.; Data curation, H.G.; Writing-original draft preparation, H.G.; Writing—review and editing, S.D.; Visualization, Q.L; Supervision, S.D.; Project administration, Y.H.; Funding acquisition, Y.H. All authors have read and agreed to the published version of the manuscript.

Funding: This research was funded by National Natural Science Foundation of China (NSFC), grant number (U1738204) and National Key R\&D Program of China, grant number (2017YFB1103002).

Conflicts of Interest: The authors declare no conflict of interest.

\section{References}

1. Cao, Z.L.; Xuan, L.; Hu, L.F.; Liu, Y.J.; Mu, Q.Q.; Li, D.Y. Investigation of optical testing with a phase-only liquid crystal spatial light modulator. Opt. Express 2005, 13, 1059-1065. [CrossRef] [PubMed]

2. Dev, K.; Singh, V.R.; Asundi, A. Full-field phase modulation characterization of liquid-crystal spatial light modulator using digital holography. Appl. Opt. 2011, 50, 1593. [CrossRef] [PubMed]

3. Konwar, S.; Boruah, B.R. Note: Current induced fluctuations in the orientation of the beam diffracted by a liquid crystal spatial light modulator. Rev. Sci. Instrum. 2017, 88, 225. [CrossRef] [PubMed]

4. Ignacio, M.; Davis, J.A.; Hernandez, T.M.; Cottrell, D.; Sand, D. Complete polarization control of light from a liquid crystal spatial light modulator. Opt. Express 2012, 20, 344.

5. Feng, F.; White, I.H.; Wilkinson, T.D. Free space communications with beam steering a two-electrode tapered laser diode using liquid-crystal SLM. J. Light Technol. 2013, 31, 2001-2007. [CrossRef]

6. Rodrigo, P.J.; Iversen, F.Q.; Hu, Q.; Pedersen, C. Diode laser lidar wind velocity sensor using a liquid-crystal retarder for non-mechanical beam-steering. Opt. Express 2014, 22, 26674-26679. [CrossRef]

7. Ivanov, A.V.; Vakulin, D.A.; Konshina, E.A. Symmetrical optical response in the hybrid-oriented twist structure of a dual-frequency nematic liquid crystal. J. Opt. Technol. 2014, 81, 130. [CrossRef]

8. Shang, Y.; Zhang, C.; Cui, N.; Guerrero, J.M. A cell-to-cell battery equalizer with zero-current switching and zero-voltage gap based on quasi-resonant LC converter and boost converter. IEEE Trans. Power Electron. 2015, 30, 3731-3747. [CrossRef]

9. Handschy, M.A. Stroboscopic microscopy of fast electro-optic switching in ferroelectric smectic $\mathrm{C}$ liquid crystals. Appl. Phys. Lett. 1982, 41, 39-41. [CrossRef]

10. Kirby, A.K.; Love, G.D. Fast, large and controllable phase modulation using dual frequency liquid crystals. Opt. Express 2004, 12, 1470-1475. [CrossRef] [PubMed]

11. Jewell, S.A.; Taphouse, T.S.; Sambles, J.R. Rapid switching in a dual-frequency hybrid aligned nematic liquid crystal cell. Appl. Phys. Lett. 2005, 87, 345. [CrossRef]

12. Wu, S.T.; Wu, C.S. Mixed-mode twisted nematic liquid crystal cells for reflective displays. Appl. Phys. Lett. 1996, 68, 1455-1457. [CrossRef]

13. Wu, S.T.; Wu, C.S. High-speed liquid-crystal modulators using transient nematic effect. J. Appl. Phys. 1989, 65, 527-534. [CrossRef]

14. Xun, X.D.; Cho, D.J.; Cohn, R.W. Spiking voltages for faster switching of nematic liquid-crystal light modulators. Appl. Opt. 2006, 45, 3134-3143. [CrossRef] [PubMed]

15. Hu, H.B.; Hu, L.F.; Peng, Z.H.; Mu, Q.Q.; Zhang, X.Y.; Liu, C.; Xuan, L. Advanced single-frame overdriving for liquid-crystal spatial light modulators. Opt. Lett. 2012, 37, 3344-3346. [CrossRef] [PubMed] 
16. Huang, Z.Q. Principle of Liquid Crystal Display; National Defence Industry Press: Beijing, China, 2006. (In Chinese)

17. Nie, X.; Xian, Y.H.; Lu, R.; Thomas, X.W.; Shin-Tson, W. Pretilt Angle Effects on Liquid Crystal Response Time. J. Disp. Technol. 2007, 3, 280-283. [CrossRef]

18. Wu, S.T.; Wu, C.S. High speed nematic liquid crystal modulators. Mol. Cryst. Liq. Cryst. 2006, $207,1-15$. [CrossRef]

19. Chen, P.C.; Chen, C.W.; Huang, Y.P.; Li, J.J. 3.5: Dual Directional Overdriving Method for Fast Response LC-lenses on Autostereoscopic 3D Display. Sid. Symp. Dig. Tech. Pap. 2012, 42, 17-20. [CrossRef]

20. O'Brien, D.C.; Faulkner, G.E.; Wilkinson, T.D.; Robertson, B.; Leyva, D.G. Design and analysis of an adaptive board-to-board dynamic holographic interconnect. Appl. Opt. 2004, 43, 3297-3305. [CrossRef] [PubMed]

21. Du, S.P.; Fu, C.Y.; Huang, Y.M.; Luo, C.X.; Wang, X.R. A new method of measure the liquid-crystal's modulating characteristic. Acta Photon. Sin. 2017, 46, 87-94. (In Chinese)

(C) 2020 by the authors. Licensee MDPI, Basel, Switzerland. This article is an open access article distributed under the terms and conditions of the Creative Commons Attribution (CC BY) license (http://creativecommons.org/licenses/by/4.0/). 